Georgian Mathematical Journal

Volume 12 (2005), Number 1, 171-179

\title{
SOME ALGEBRAIC AND GEOMETRIC STRUCTURES ON POISSON MANIFOLDS
}

\author{
ZAZA TEVDORADZE
}

\begin{abstract}
In this paper we study some algebraic properties of harmonic forms on Poisson manifolds. It is well known that in the classical case (on Riemannian manifolds) the product of harmonic forms is not harmonic. Here we describe the algebraic and analytical mechanisms explaining this fact. We also obtain a condition under which the product of de Rham cohomology classes, which includes harmonic representatives, can be represented by a harmonic form.
\end{abstract}

2000 Mathematics Subject Classification: 53D17, 58A14.

Key words and phrases: Poisson manifolds, symplectic manifold, harmonic form, canonical homology.

\section{INTRODUCTION}

The main aim of this paper is to study some algebraic properties of harmonic forms on Poisson manifolds.

It is well known that $\delta$ operator can be introduced on a Poisson manifold $M^{n}$ ([1], [2]) (by analogy with the classical Hodge theory on Riemannian manifolds) and in case when $M^{n}$ is symplectic manifold, the notion of harmonic forms was introduced by Brylinski ([1]).

In this paper we introduce the notion of harmonic form on Poisson manifold and examine algebraic and analytic obstructions to the existence of a multiplicative structure in the space of harmonic forms (Corollary 2.2, Theorem 2.4, Corollary 2.5). As a corollary we will allocate the class of harmonic forms which is closed with respect to the exterior product (Corollary 2.3). In this section we also have established one simple property of harmonic forms with respect to compatible Poisson structures (Theorem 2.7).

J.-L. Brylinski conjectured that on a compact symplectic manifold every de Rham cohomology class contains a harmonic representative [1]. In the same paper this conjecture was proved in case when $M^{n}$ is Kähler manifold. In ([3]) O. Mathieu disproved Brylinski's conjecture. About this question one can also see D. Yan's paper [4]. As the examples which are constructed in these papers are rather complicated, at the end of the paper we give a simple example of compact Poisson manifold which disproves Brylinski's conjecture (obviously in more weak case, where $M^{n}$ is Poisson manifold).

The paper is organized as follows. In the first section some well-known basic definitions and facts are given; one can see them, for example, in [7]. 
In Section 2 we consider the notion of harmonic form on a Poisson manifold and construct the cohomology groups which contain obstructions to existence of a multiplicative structure in the space of harmonic forms. In this section we also establish one simple property of harmonic forms with respect to compatible Poisson structures and show that not every cohomology class on a Poisson manifold can be represented by harmonic forms.

\section{Some Basic Definitions and Facts}

1.1. The Schouten-Nijenhuis bracket. Let $M^{n}$ be a smooth real manifold, and let $\mathcal{A}=C^{\infty}\left(M^{n}\right)$ be the algebra of smooth real-valued functions on $M^{n}$.

Also, let $\bigwedge\left(M^{n}\right)=\bigoplus_{i=0}^{n} \bigwedge_{i}\left(M^{n}\right)$ be the contravariant Grassmann algebra on $M^{n}$.

It is well known that for arbitrary $X \in \bigwedge_{1}\left(M^{n}\right)$ there is a well defined Lie derivative operator $L_{X}$ which, in particular, acts on $\bigwedge_{i}\left(M^{n}\right)$ by

$$
\left(L_{X} Y\right)\left(x_{0}\right)=\left.\frac{d}{d t}\right|_{t=0}\left\{\exp (-t X)_{*} Y\left(\exp \left(t X\left(x_{0}\right)\right)\right)\right\}
$$

where $x_{0} \in M^{n}, Y \in \bigwedge_{i}\left(M^{n}\right)$. It is well known that $L_{X} Y$ coincides with $[X, Y]$ when $i=1$. Thus, for arbitrary vector fields $X_{1}, X_{2}, \ldots, X_{p}$ and $Y \in \bigwedge_{i}\left(M^{n}\right)$, it is natural to define the operation

$$
\left[X_{1} \wedge \cdots \wedge X_{p}, Y\right]=(-1)^{p+1} \sum_{i=1}^{p}(-1)^{i+1} X_{1} \wedge \cdots \wedge \hat{X}_{i} \wedge \cdots \wedge X_{p} \wedge L_{X_{i}} Y
$$

where $\wedge$ denotes that the corresponding factor is omitted.

There exists a unique $\mathbb{R}$-bilinear map, defined on $\bigwedge\left(M^{n}\right) \times \bigwedge\left(M^{n}\right)$, with values in $\bigwedge\left(M^{n}\right)$, called the Schouten-Nijenhuis bracket (SNB) and denoted by $[$,$] and satisfying the following properties:$

(a) for $f, g \in \mathcal{A},[f, g]=0$,

(b) for a vector field $X \in \bigwedge_{1}\left(M^{n}\right)$ and a multi-vector field $Y \in \bigwedge_{q}\left(M^{n}\right)$, $[X, Y]$ coincides with the Lie derivation $L_{X} Y$,

(c) for $A \in \bigwedge_{p}\left(M^{n}\right), B \in \bigwedge_{q}\left(M^{n}\right)$,

$$
[A, B]=-(-1)^{(p-1)(q-1)}[B, A],
$$

(d) for $A \in \bigwedge_{p}\left(M^{n}\right), B \in \bigwedge_{q}\left(M^{n}\right)$ and $C \in \bigwedge_{r}\left(M^{n}\right)$,

$$
[A, B \wedge C]=[A, B] \wedge C+(-1)^{(p-1) q} B \wedge[A, C] .
$$

For the proof of these properties one can see, for example, [1] or [7].

From (1.3) and (1.4) we can conclude that the SNB is a local type bracket (its values in an open subset of $M^{n}$ depend only on the values of multi-vector fields on that open subset) and defines a bilinear mapping $\bigwedge_{p}\left(M^{n}\right) \times \bigwedge_{q}\left(M^{n}\right) \rightarrow$ $\bigwedge_{p+q-1}\left(M^{n}\right)$ by the condition

$$
\begin{aligned}
{\left[X_{1} \wedge \cdots \wedge X_{p}, Y_{1} \wedge \cdots \wedge Y_{q}\right]=} & \sum_{t, s}(-1)^{p+1+t+s} X_{1} \wedge \cdots \wedge \hat{X}_{s} \wedge \cdots \wedge X_{p} \\
& \wedge\left[X_{s}, Y_{t}\right] \wedge Y_{1} \wedge \cdots \wedge \hat{Y}_{t} \wedge \cdots \wedge Y_{q}
\end{aligned}
$$


where $X_{1}, \ldots, X_{p}, Y_{1}, \ldots, Y_{q}$ are vector fields on $M^{n}$. From formulae (1.3),(1.4), (1.5) we can obtain the following graded Jacobi identity:

$$
\begin{aligned}
(-1)^{(p-1)(r-1)}[[A, B], C] & +(-1)^{(q-1)(p-1)}[[B, C], A] \\
& +(-1)^{(q-1)(r-1)}[[C, A], B]=0
\end{aligned}
$$

where $A \in \bigwedge_{p}\left(M^{n}\right), B \in \bigwedge_{q}\left(M^{n}\right)$ and $C \in \bigwedge_{r}\left(M^{n}\right)$.

Remark 1.1. Identities (1.3) and (1.6) imply that the graded vector space $\bigwedge\left(M^{n}\right)=\bigoplus_{i=0}^{n} \bigwedge_{i}\left(M^{n}\right)$, with the Schouten-Nijenhuis bracket as a composition law, is a graded Lie algebra. In order to have a simple rule for composing the degrees, one has to state that the "Lie degree" of a homogeneous multi-vector field $A \in \bigwedge_{p}\left(M^{n}\right)$ is $p-1$. Of course, the Lie degree of $A \in \bigwedge_{p}\left(M^{n}\right)$ should not be confused with its ordinary degree (so-called exterior degree) which is $p$.

Remark 1.2. According to Remark 1.1, the graded Jacobi identity can be written in other forms, in which its meaning is clearer than in the form (1.6). For each $p \in \overline{0, n}$, let us define the mapping

$$
a d_{-}^{p}: \bigwedge_{p}\left(M^{n}\right) \rightarrow \mathcal{H o m}\left(\bigwedge\left(M^{n}\right)\right)
$$

by the formula $a d_{A}^{p}(B)=[A, B]$, where $A \in \bigwedge_{p}\left(M^{n}\right), B \in \bigwedge\left(M^{n}\right)$ and $\mathcal{H o m}\left(\bigwedge\left(M^{n}\right)\right)$ denotes the space of endomorphisms of the linear space $\bigwedge\left(M^{n}\right)$. These mappings can be extended by linearity on the space $\bigwedge\left(M^{n}\right)$ and we denote it by

$$
a d_{-}: \bigwedge\left(M^{n}\right) \rightarrow \mathcal{H o m}\left(\bigwedge\left(M^{n}\right)\right) .
$$

Then, as we know, the values of the operator $a d_{-}^{p}$ are graded linear endomorphisms of $\bigwedge\left(M^{n}\right)$ and thus the graded Jacobi identity (1.6) can be written in a more acceptable form (from the viewpoint of the theory of Lie algebras)

$$
a d_{A}[B, C]=\left[a d_{A} B, C\right]+(-1)^{(p-1)(q-1)}\left[B, a d_{A} C\right]
$$

or as

$$
a d_{[A, B]}=a d_{A} \circ a d_{B}-(-1)^{(p-1)(q-1)} a d_{B} \circ a d_{A},
$$

where $A \in \bigwedge_{p}\left(M^{n}\right), B \in \bigwedge_{q}\left(M^{n}\right)$ and $C \in \bigwedge_{r}\left(M^{n}\right)$. Equation (1.9) has a clear meaning: the graded endomorphism $a d_{A}$, of degree $p-1$, is a derivation of the graded Lie algebra $\bigwedge\left(M^{n}\right)$ with the SNB as a composition law (the degree of $B \in \bigwedge_{q}\left(M^{n}\right)$ considered here being its Lie degree $q-1$ rather than its exterior degree $q$ ). Equation (1.10) means that the endomorphism $a d_{[A, B]}$ of degree $p+q-2$, is the graded commutator of the endomorphisms $a d_{A}$ (of degree $p-1$ ) and $a d_{B}$ (of degree $q-1$ ).

1.2. Lichnerowicz derivation. Let $M^{n}$ be a smooth Poisson manifold, i.e., there exists a $(2,0)$-type contravariant antisymmetric tensor field $G \in \bigwedge_{2}\left(M^{n}\right)$ on $M^{n}$ such that $[G, G]=0$, where [, ] denotes the Schouten-Nijenhuis bracket. Then we can define the Poisson bracket of the functions $f$ and $g$ from $\mathcal{A}$ by the formula

$$
\{f, g\}=i_{G}(d f \wedge d g)
$$


satisfying the following properties:

a)

$$
\{f, g\}=-\{g, f\}
$$

$$
\begin{gathered}
\{f, g \cdot h\}=\{f, g\} \cdot h+g \cdot\{f, h\} \\
\{\{f, g\}, h\}+\{\{g, h\}, f\}+\{\{h, f\}, g\}=0,
\end{gathered}
$$

where $f, g, h \in \mathcal{A}$.

From the property b) it is clear that, for a given function $H \in \mathcal{A}$, the mapping $f \rightarrow\{H, f\}$ defines the differentiation of the algebra $\mathcal{A}$ and so there exists a (uniquely defined) vector field $V_{H}$ on $M^{n}$ such that $\{H, g\}=V_{H} g$ for all $g \in \mathcal{A}$. The vector field $V_{H}$ is called the Hamiltonian vector field of $H$. It is easy to check that $V_{\{f, g\}}=\left[V_{f}, V_{g}\right]$. Further by $d_{G}$ we denote the operator $a d_{G}$. From (1.10) it follows that the operator $d_{G}: \bigwedge_{p}\left(M^{n}\right) \rightarrow \bigwedge_{p+1}\left(M^{n}\right)$ is nilpotent, more precisely, $d_{G}{ }^{2}=0$. From (1.4) and (1.6) we have

$$
d_{G}(B \wedge C)=d_{G}(B) \wedge C+(-1)^{q} B \wedge d_{G}(C)
$$

and

$$
d_{G}[B, C]=\left[d_{G} B, C\right]+(-1)^{q-1}\left[B, d_{G} C\right],
$$

where $B \in \bigwedge_{q}\left(M^{n}\right)$ and $C \in \bigwedge_{r}\left(M^{n}\right)$. The cohomology of the complex $\left(\bigwedge_{*}\left(M^{n}\right), d_{G}\right)$ is called the Lichnerowicz-Poisson cohomology and we denote it by $H_{\mathcal{L P}}{ }^{*}\left(M^{n}\right)$ (for this see [5]).

The Poisson structure defines a very important polar mapping $P: T^{*} M^{n} \rightarrow$ $T M^{n}$ by the formula $P(\alpha)=G\left\lfloor\alpha\right.$, where $\alpha \in \bigwedge^{1}\left(M^{n}\right)$ is a differential 1-form on $M^{n}$.

1.2.1. Coordinate expressions. Let $\left(x^{i}\right)_{i=\overline{1, n}}$ be a local coordinate system in the neighborhood $\mathcal{U} \subset M^{n}$, and let the restriction of the $(2,0)$-contravariant tensor field $G$ on $\mathcal{U}$ be represented by the formula $G=\frac{1}{2 !} G^{i j} \frac{\partial}{\partial x^{i}} \wedge \frac{\partial}{\partial x^{j}}$. Then if $f, g \in \mathcal{A}$ are smooth functions on $M^{n}$, from (1.11) we obtain

$$
\left.\{f, g\}\right|_{\mathcal{U}}=G^{i j} \frac{\partial f}{\partial x^{i}} \cdot \frac{\partial g}{\partial x^{j}}
$$

and

$$
\left.V_{f}\right|_{\mathcal{U}}=G^{i j} \frac{\partial f}{\partial x^{i}} \frac{\partial}{\partial x^{j}} .
$$

We have $\left.d_{G}(f)\right|_{\mathcal{U}}=-G^{i j} \frac{\partial f}{\partial x^{i}} \frac{\partial}{\partial x^{j}}=-\left.V_{f}\right|_{\mathcal{U}}$.

If $B=\frac{1}{q !} B^{j_{1} \cdots j_{q}} \frac{\partial}{\partial x^{j_{1}}} \wedge \cdots \wedge \frac{\partial}{\partial x^{j q}}$ is a restriction of the multi-vector field on $M^{n}$, then from (1.12) we conclude

$$
\begin{aligned}
d_{G}(B)= & -\frac{1}{q !} G^{u i} \frac{\partial B^{j_{1} \cdots j_{q}}}{\partial x^{u}} \frac{\partial}{\partial x^{i}} \wedge \frac{\partial}{\partial x^{j_{1}}} \wedge \cdots \wedge \frac{\partial}{\partial x^{j_{q}}} \\
& -\frac{1}{2(q-1) !} B^{u j_{2} \cdots j_{q}} \frac{\partial G^{i j}}{\partial x^{u}} \frac{\partial}{\partial x^{i}} \wedge \frac{\partial}{\partial x^{i}} \wedge \frac{\partial}{\partial x^{j_{2}}} \wedge \cdots \wedge \frac{\partial}{\partial x^{j_{q}}} .
\end{aligned}
$$


If $\left.\alpha\right|_{\mathcal{U}}=a_{i} d x^{i}$ is the restriction of an arbitrary one-dimensional differential form on $\mathcal{U}$, it is easy to verify that

$$
P(\alpha)=G^{i j} a_{i} \frac{\partial}{\partial x^{j}}
$$

and so we conclude that

$$
P(d H)=V_{H}=-d_{G}(H)
$$

for all $H \in \mathcal{A}$.

1.3. De Rham cohomology versus Lichnerowicz-Poisson cohomology. By analogy with the SNB on $\Lambda\left(M^{n}\right)$, one can define the $\mathbb{R}$-bilinear bracket $\{\cdot, \cdot\}^{G}$ on $\Omega\left(M^{n}\right)=\bigoplus_{i=0}^{n} \bigwedge^{i}\left(M^{n}\right)$, whose restriction on

$$
\{\cdot, \cdot\}^{G}: \bigwedge^{p}\left(M^{n}\right) \times \bigwedge^{q}\left(M^{N}\right) \rightarrow \bigwedge^{p+q-1}\left(M^{n}\right)
$$

satisfies the following two conditions (see [7]):

i) the restriction of $\{,\}^{G}$ on $\bigwedge^{1}\left(M^{n}\right) \times \bigwedge^{1}\left(M^{n}\right)$ defines a Lie structure on $\bigwedge^{1}\left(M^{n}\right)$ such that $P$ is a homomorphism of Lie algebras;

ii) $\left\{\alpha_{1} \wedge \cdots \wedge \alpha_{k}, \beta_{1} \wedge \cdots \wedge \beta_{q}\right\}^{G}=\sum_{t, s}(-1)^{k+1+t+s} \alpha_{1} \wedge \cdots \wedge \hat{\alpha_{s}} \cdots \wedge \alpha_{k}$

$$
\wedge\left\{\alpha_{s}, \beta_{t}\right\}^{G} \wedge \beta_{1} \wedge \cdots \wedge \hat{\beta}_{t} \cdots \wedge \beta_{q}
$$

So, $\Omega\left(M^{n}\right)$ is also a graded Lie algebra with $\{,\}^{G}$ as a composition law. From the conditions i) and ii) we can conclude that the mapping $\wedge^{*}(P): \wedge^{*}\left(M^{n}\right) \rightarrow$ $\bigwedge_{*}\left(M^{n}\right)$ is a homomorphism of graded Lie algebras, where $\wedge^{*}(P)$ is deduced from $P$ as an exterior product of $P$.

1.3.1. Coordinate expression. Let $\left(x^{i}\right)_{i=\overline{1, n}}$ be the local coordinate system in the neighborhood $\mathcal{U} \subset M^{n}$, and let the restriction of the (2,0)-contravariant tensor field $G$ on $\mathcal{U}$ be represented by the formula $G=\frac{1}{2 !} G^{i j} \frac{\partial}{\partial x^{i}} \wedge \frac{\partial}{\partial x^{j}}$. Let $\left.\alpha\right|_{\mathcal{U}}=\frac{1}{p !} \alpha_{i_{1} \ldots i_{p}} d x^{i_{1}} \wedge \cdots \wedge d x^{i_{p}}$ and $\left.\beta\right|_{\mathcal{U}}=\frac{1}{q !} \beta_{j_{1} \ldots j_{q}} d x^{j_{1}} \wedge \cdots \wedge d x^{j_{q}}$ be the restriction of differential forms $\alpha \in \wedge^{p}\left(M^{n}\right)$ and $\beta \in \wedge^{q}\left(M^{n}\right)$ on $\mathcal{U}$; then

$$
\begin{aligned}
\left\{\left.\alpha\right|_{\mathcal{U}},\left.\beta\right|_{\mathcal{U}}\right\}_{i_{1} \ldots i_{p+q-1}}^{G} & =\frac{(-1)^{p}}{(p-1) ! q !} \varepsilon_{i_{1} \ldots i_{p+q-1}}^{j_{2} \ldots j_{p} t_{1} \ldots t_{q}} G^{u s} \alpha_{s j_{2} \ldots j_{p}} \cdot \frac{\partial}{\partial x^{u}}\left(\beta_{t_{1} \ldots t_{q}}\right) \\
& +\frac{1}{(q-1) ! p !} \varepsilon_{i_{1} \ldots i_{p+q-1}}^{j_{1} \ldots j_{p} t_{2} \ldots t_{q}} G^{u s} \frac{\partial}{\partial x^{u}}\left(\alpha_{j_{1} \ldots j_{p}}\right) \cdot \beta_{s t_{2} \ldots t_{q}} \\
& +\frac{1}{(q-1) !(p-1) !} \varepsilon^{u j_{1} \ldots i_{p+q-1}} \frac{1}{\partial x^{u}}\left(G^{s l}\right) \alpha_{s j_{2} \ldots j_{p}} \cdot \beta_{l t_{2} \ldots t_{q}},
\end{aligned}
$$

where $\varepsilon_{\ldots}^{\ldots}$ is the Kronecker multi-index, i.e., 1 (or -1) if the upper indices are an even (odd) permutation of lower indices, and 0 otherwise. 
One can verify that $\wedge(P)=\bigoplus_{i=0}^{n} \wedge_{i}(P)$ induces an antihomomorphism between de Rham and Lichnerowicz-Poisson differential complexes ([7]), i.e.,

$$
\wedge_{p+1}(P) \circ d=-d_{G} \circ \wedge_{p}(P),
$$

where $\wedge_{0}(P)=i d$ and $\wedge_{1}(P)=P$.

So the polar mapping $P$ induces homomorphisms

$$
\wedge_{*}(P): H_{\mathcal{D R}}{ }^{*}\left(M^{n}\right) \rightarrow H_{\mathcal{L P}}{ }^{*}\left(M^{n}\right)
$$

which are obviously isomorphisms when $P$ is an isomorphism (it is so if and only if $M^{n}$ is a symplectic manifold).

1.4. Canonical homologies. One can verify that on $\bigwedge^{1}\left(M^{n}\right)$ aforementioned bracket $\{,\}^{G}$ has the form

$$
\{f d H, g d Q\}^{G}=f g d\{H, Q\}-f\{g, H\} d Q+g\{f, Q\} d H,
$$

where $f, g, H, Q \in \mathcal{A}$. In a more invariant way, for arbitrary 1-forms $\alpha$ and $\beta$ we have

$$
\{\alpha, \beta\}^{G}=L_{P(\alpha)} \beta-L_{P(\beta)} \alpha+d(G\lfloor(\alpha \wedge \beta)) .
$$

J.-L. Koszul (see, e.g., [2], [5]) introduced a differential $\delta_{*}: \bigwedge^{*}\left(M^{n}\right) \rightarrow$ $\bigwedge^{*-1}\left(M^{n}\right)$ defined as $\delta_{*}=i_{G} \circ d^{*}-d^{*-2} \circ i_{G}$, which satisfies condition $\delta^{2}=0$. In [1] this differential is expressed by the formula

$$
\begin{aligned}
\delta\left(f_{0} d f_{1} \wedge \cdots \wedge d f_{k}\right)= & \sum_{1 \leq i \leq k}(-1)^{i+1}\left\{f_{0}, f_{i}\right\} d f_{1} \wedge \cdots \wedge \hat{d f_{i}} \wedge \cdots \wedge d f_{k} \\
& +\sum_{1 \leq i<j \leq k}(-1)^{i+j} f_{0} d\left\{f_{i}, f_{j}\right\} \wedge d f_{1} \wedge \cdots \wedge \hat{d f_{i}} \\
& \wedge \cdots \wedge \hat{d f}_{j} \wedge \cdots \wedge d f_{k} .
\end{aligned}
$$

So, for a Poisson manifold $\left(M^{n}, G\right)$, there is a canonical complex $\left(\bigwedge^{*}\left(M^{n}\right), \delta_{*}\right)$ whose homology groups are denoted by $H_{*}^{c a n}\left(M^{n}\right)$ and called canonical homologies of $\left(M^{n}, G\right)$.

The canonical complex $\left(\bigwedge^{*}\left(M^{n}\right), \delta_{*}\right)$ is not a differential complex. The declination of $\delta$ from the differentiability can be expressed by the bracket $\{,\}^{G}$. More precisely, we have the following proposition (the proof can be found in $[7])$.

Proposition 1.3. If $\alpha \in \bigwedge^{p}\left(M^{n}\right)$ and $\beta \in \bigwedge^{q}\left(M^{n}\right)$, then

$$
(-1)^{p+1}\{\alpha, \beta\}^{G}=\delta \alpha \wedge \beta+(-1)^{p} \alpha \wedge \delta \beta-\delta(\alpha \wedge \beta) .
$$

\section{Harmonic Forms on Poisson Manifolds}

From (1.25), by direct calculations we can conclude that the operators $d^{i-1} \delta_{i}+$ $\delta_{i+1} d^{i}$ are identically zero, so, on Poisson manifolds, "Laplacian" operators are not defined as they are on Riemannian manifolds.

Similarly to Brylinski's definition of the harmonic forms on symplectic manifolds we can define harmonic forms on Poisson manifolds. 
Definition 2.1. A differential form $\alpha \in \Lambda^{*}\left(M^{n}\right)$ on the Poisson manifold $\left(M^{n}, G\right)$ is called harmonic if it satisfies the condition $d \alpha=\delta \alpha=0$.

It is well known that even in the classical case the product of harmonic forms is not a harmonic form.

From Proposition 1.3 we have the following corollary.

Corollary 2.2. If $\alpha$ and $\beta$ are harmonic differential forms on a Poisson manifold $M^{n}$, then $\alpha \wedge \beta$ is a harmonic differential form if and only if $\{\alpha, \beta\}^{G}=$ 0 .

Now let us consider the regular Poisson manifold $\left(M^{n}, G\right)$, i.e., the $\operatorname{rank}(G)=$ const, and fix on it some torsionless connection $\nabla$. For the existence of a torsionless connection on such Poisson manifolds see $([7])$. If $\gamma_{j k}^{i}$ denotes the coefficients of connection $\nabla$ in local coordinates, then for the differential $p$-form $\alpha$ we have

$$
\nabla_{u} \alpha_{i_{1} \cdots i_{p}}=\frac{\partial}{\partial x^{u}} \alpha_{i_{1} \cdots i_{p}}-\gamma_{i_{1} u}^{j} \alpha_{j i_{2} \cdots i_{p}}-\cdots-\gamma_{i_{p} u}^{j} \alpha_{i_{1} \cdots i_{p-1} j} .
$$

Taking into account this equality and the fact that $\{\alpha, \beta\}_{G i_{1} \cdots i_{p+q-1}}$ is antisymmetric tensor, we can rewrite (1.20) as follows:

$$
\begin{aligned}
\left\{\left.\alpha\right|_{\mathcal{U}},\left.\beta\right|_{\mathcal{U}}\right\}_{i_{1} \ldots i_{p+q-1}}^{G} & =\frac{(-1)^{p}}{(p-1) ! q !} \varepsilon_{i_{1} \ldots i_{p+q-1}}^{j_{2} \ldots j_{p} t_{1} \ldots t_{q}} G^{u s} \alpha_{s j_{2} \ldots j_{p}} \cdot \nabla_{u}\left(\beta_{t_{1} \ldots t_{q}}\right) \\
& +\frac{1}{(q-1) ! p !} \varepsilon_{i_{1} \ldots i_{p+q-1}}^{j_{1} \ldots j_{p} t_{2} \ldots t_{q}} G^{u s} \nabla_{u}\left(\alpha_{j_{1} \ldots j_{p}}\right) \cdot \beta_{s t_{2} \ldots t_{q}} \\
& +\frac{1}{(q-1) !(p-1) !} \varepsilon_{i_{1} \ldots i_{p+q-1}}^{u j_{2} \ldots j_{p} t_{2} \ldots t_{q}} \nabla_{u}\left(G^{s l}\right) \alpha_{s j_{2} \ldots j_{p}} \cdot \beta_{l t_{2} \ldots t_{q}} .
\end{aligned}
$$

Corollary 2.3. Let $\nabla$ be a Poisson connection on the Poisson manifold $M^{n}$ of constant rank, and let $\alpha$ and $\beta$ be parallel harmonic differential forms with respect to the connection $\nabla$, then $\alpha \wedge \beta$ is also a harmonic differential form.

Now let us pose the following question: if $\alpha \in \Lambda^{i}\left(M^{n}\right)$ and $\beta \in \Lambda^{i}\left(M^{n}\right)$ are the harmonic forms on the Poisson manifold $\left(M^{n}, G\right)$, then does there exist a harmonic representative of the class $[\alpha \wedge \beta]$ in the de Rham cohomology?

The answer on this question, in general, is negative: we construct the cohomology groups which contain the obstruction to this fact. Below (see Example 2.9) we show that, in general, not every de Rham cohomology class can be represented by the harmonic forms.

Let $B^{i}$ denotes the $\operatorname{Im} d^{i-1}$, then as $d^{i-1} \delta_{i}+\delta_{i+1} d^{i}=0$, the chain complex $\left(B^{*}, \delta_{*}\right)$ is defined correctly and the homology of this complex we denote as $H_{*}^{T}\left(M^{n}\right)$.

Theorem 2.4. Let $\alpha \in \Lambda^{i}\left(M^{n}\right)$ and $\beta \in \Lambda^{i}\left(M^{n}\right)$ be harmonic forms on the Poisson manifold $\left(M^{n}, G\right)$. Then the de Rham cohomology class of the form $\alpha \wedge \beta$ can be represented by a harmonic form if and only if $\left[\{\alpha, \beta\}^{G}\right]$ is a trivial homology class in $H_{i+j-1}^{T}\left(M^{n}\right)$ homology group. 
Proof. Let us assume that the de Rham homology class $\alpha \wedge \beta$ can be represented by a harmonic form $\gamma \in \Lambda_{i+j}\left(M^{n}\right)$, i.e., $\delta \gamma=d \gamma=0$ and there is a form $a \in \Lambda_{i+j-1}\left(M^{n}\right)$ such that $\gamma=\alpha \wedge \beta+d a$. Then $\delta \gamma=\delta \alpha \wedge \beta+(-1)^{i} \alpha \wedge$ $\delta \beta+(-1)^{i}\{\alpha, \beta\}_{G}+\delta d a=(-1)^{i}\{\alpha, \beta\}_{G}+\delta d a=0$. From this equation we get $\{\alpha, \beta\}_{G}=\delta d\left((-1)^{i+1} a\right)$, which means that $\left[\{\alpha, \beta\}_{G}\right]=0$ in the homology group $H_{i+j-1}^{T}\left(M^{n}\right)$. Now let $\{\alpha, \beta\}_{G}=\delta d a$ for some $i+j-1$ dimensional differential form $a \in \Lambda_{i+j-1}\left(M^{n}\right)$. Then $\delta(\alpha \wedge \beta)=(-1)^{i}\{\alpha, \beta\}_{G}=(-1)^{i} \delta d a$. We denote the $i+j$ dimensional differential form $\alpha \wedge \beta+(-1)^{i+1} d a$ by $\gamma$. It is clear that $d \gamma=0$ and $\delta \gamma=(-1)^{i} \delta d a+(-1)^{i+1} \delta d a=0$, i.e., $\gamma$ is a harmonic form and $[\gamma]=[\alpha \wedge \beta]$ in the de Rham cohomology group $H^{i+j}\left(M^{n}\right)$.

Corollary 2.5. If any cohomology class in de Rham cohomologies can be represented by a harmonic form, then for arbitrary differential forms $\alpha \in \Lambda^{i}\left(M^{n}\right)$ and $\beta \in \Lambda^{i}\left(M^{n}\right),\left[\{\alpha, \beta\}^{G}\right]=0$ in T-homologies. In particular, this occurs when $M^{n}$ is the Kähler manifold.

As a $d^{i-1} \delta_{i}+\delta_{i+1} d^{i}=0$, it is clear that $\hat{\delta}_{i}=(-1)^{i} \delta_{i}$ defines homomorphisms between de Rham cohomologies $\hat{\delta}_{i}^{*}: H^{i}\left(M^{n}\right) \rightarrow H^{i-1}\left(M^{n}\right)$. It is also clear that the necessary condition for $[\alpha] \in H^{i}\left(M^{n}\right)$ could be represented by a harmonic form such that $\hat{\delta}_{i}^{*}([\alpha])=0$, i.e., if in every cohomology class there exists a harmonic form, then homomorphisms $\hat{\delta}_{i}^{*}$ are identically zero. Below (in example $2.9)$ we will see that this condition is not sufficient.

Now let us consider two compatible Poisson structures on manifold $M^{n}$.

Definition 2.6. Two Poisson structures $G_{1}$ and $G_{2}$ on the manifold $M^{n}$ are called compatible if $\left[G_{1}, G_{2}\right]=0$, i.e., $G_{1}$ and $G_{2}$ are compatible if and only if any linear combination $\lambda_{1} G_{1}+\lambda_{2} G_{2}$ defines the Poisson structure on $M^{n}$.

Theorem 2.7. If $G^{\prime}$ and $G^{\prime \prime}$ are compatible Poisson structures on the manifold $M^{n}$, then if $\alpha \in \Lambda^{i}\left(M^{n}\right)$ is harmonic form corresponding to the structure $G^{\prime}$, then $\delta^{\prime \prime} \alpha$ is also a harmonic form corresponding to the structure $G^{\prime}$, where $\delta^{\prime \prime}$ is the operator $\delta$ defined by the structure $G^{\prime \prime}$.

Proof. One can immediately verify the formula $i_{\left[G^{\prime}, G^{\prime \prime}\right]}=i_{G^{\prime}} d i_{G^{\prime \prime}}+i_{G^{\prime \prime}} d i_{G^{\prime}}-$ $i_{G^{\prime}} i_{G^{\prime \prime}} d-d i_{G^{\prime} \wedge G^{\prime \prime}}$. According to this formula and the facts that $d \alpha=\delta^{\prime} \alpha=0$, we obtain $i_{G^{\prime}} \delta^{\prime \prime} \alpha=d i_{G^{\prime \prime}} i_{G^{\prime}} \alpha$. From the latter formula we get $\delta^{\prime}\left(\delta^{\prime \prime} \alpha\right)=0$. It is also clear that $d\left(\delta^{\prime \prime} \alpha\right)=-\delta^{\prime \prime} d \alpha=0$.

Example 2.8. In this example we show that if $G^{\prime}$ and $G^{\prime \prime}$ are compatible Poisson structures on the manifold $M^{n}$ and $\alpha \in \Lambda^{i}\left(M^{n}\right)$ is a harmonic form corresponding to the structure $G^{\prime}$, then it is not necessary that it be harmonic corresponding to the structure $G^{\prime \prime}$. Let us consider two compatible Poisson structure $G^{\prime}=\frac{\partial}{\partial x^{1}} \wedge \frac{\partial}{\partial x^{2}}$ and $G^{\prime \prime}=\frac{\partial}{\partial x^{3}} \wedge \frac{\partial}{\partial x^{4}}$ on the manifold $\mathbb{R}^{4}$, where $\left(x^{1}, x^{2}, x^{3}, x^{4}\right)$ is the coordinate system in $\mathbb{R}^{4}$. It is clear that the form $\alpha=x^{3} d x^{3} \wedge d x^{4}$ is harmonic under the structure $G^{\prime}$, but $\delta^{\prime \prime} \alpha=d i_{G^{\prime \prime}} \alpha=d x^{3} \neq 0$.

Example 2.9. Let us consider the flat torus $T^{3}$ and let $\varphi, \psi, \vartheta \in[0,2 \pi]$ is the local coordinate system on $T^{3}$. $G=\cos \psi \frac{\partial}{\partial \varphi} \wedge \frac{\partial}{\partial \psi}$ defines the Poisson structure 
on $T^{3}$. Let $\alpha=f d \varphi \wedge d \psi+g d \psi \wedge d \vartheta+h d \varphi \wedge d \vartheta$ be a harmonic 2-form on $T^{3}$, where $f, g, h \in C^{\infty}\left(T^{3}\right)$. Then $d \alpha=0$ implies, that $\frac{\partial f}{\partial \vartheta}+\frac{\partial g}{\partial \varphi}-\frac{\partial h}{\partial \psi}=0$. From the equation $\delta \alpha=0$ we get $f \cos \psi=k=$ const. The latter equation implies $f=\frac{k}{\cos \psi}$ and as the function $f$ is continuous on $T^{3}$, we conclude that $k=0$ and so $f=0$. Summarizing these facts, we conclude that every harmonic form on the above-mentioned Poisson manifold has the form $\alpha=g d \psi \wedge d \vartheta+h d \varphi \wedge d \vartheta$, where $\frac{\partial g}{\partial \varphi}=\frac{\partial h}{\partial \psi}$. It is well known that $H^{2}\left(T^{3}\right)=\left(H^{1}\left(T_{\varphi}^{1}\right) \otimes H^{1}\left(T_{\psi}^{1}\right) \otimes H^{0}\left(T_{\vartheta}^{1}\right)\right)$ $\oplus\left(H^{1}\left(T_{\varphi}^{1}\right) \otimes H^{0}\left(T_{\psi}^{1}\right) \otimes H^{1}\left(T_{\vartheta}^{1}\right)\right) \oplus\left(H^{0}\left(T_{\varphi}^{1}\right) \otimes H^{1}\left(T_{\psi}^{1}\right) \otimes H^{1}\left(T_{\vartheta}^{1}\right)\right)=R \oplus R \oplus R$, where $T_{\varphi}^{1}, T_{\psi}^{1}, T_{\vartheta}^{1}$ defines coordinate circles in the torus. It is clear that the harmonic forms $\alpha=g d \psi \wedge d \vartheta+h d \varphi \wedge d \vartheta$ cannot represent cohomology classes from the item $H^{1}\left(T_{\varphi}^{1}\right) \otimes H^{1}\left(T_{\psi}^{1}\right) \otimes H^{0}\left(T_{\vartheta}^{1}\right)$.

So Example 2.9 shows that in the general case not every cohomology class can be represented by harmonic forms.

\section{REFERENCES}

1. J.-L. Brylinski, A differential complex for Poisson manifolds. J. Differential Geom. 28(1988), No. 1, 93-114.

2. J.-L. Koszul, Crochet de Schouten-Nijenhuis et cohomologie. The mathematical heritage of Élie Cartan (Lyon, 1984). Astérisque 1985, Numero Hors Serie, 257-271.

3. O. Mathieu, Harmonic cohomology classes of symplectic manifolds. Comment. Math. Helv. 70(1995), No. 1, 1-9.

4. D. YAN, Hodge structure on symplectic manifolds. Adv. Math. 120(1996), No. 1, 143-154.

5. A. Lichnerowicz, Global theory of connections and holonomy groups. (Translated from the French) Noordhoff International Publishing, Leiden, 1976.

6. A. Lichnerowicz, Les variétés de Poisson et leurs algèbres de Lie associées. J. Differential Geometry 12(1977), No. 2, 253-300.

7. I. Vaisman, Lectures on the geometry of Poisson manifolds. Progress in Mathematics, 118.Birkhäuser Verlag, Basel, 1994.

(Received 4.05.2004; revised 15.12.2004)

Author's address:

Department of Mechanics and Mathematics

I. Javakhishvili Tbilisi State University

2, University St., Tbilisi 0143, Georgia

E-email: zatevd@geo.net.ge 